\title{
Avances y desafíos en las vacunas el para control de la pandemia por COVID-19
}

\author{
Advances and challenges in vaccines to control the COVID-19 pandemic
}

\author{
Caroline R. Weinstein-Oppenheimer ${ }^{1 *}$, Max Andresen², Raúl Corrales ${ }^{3}$, Jorge Gallardo ${ }^{4}$, Viviana Noriega ${ }^{5}$, Eliana Sánchez ${ }^{6}$
}

Desde diciembre de 2020 a la fecha se ha observado un notable avance en la diversidad de vacunas para combatir la COVID-19 que han recibido la aprobación para uso de emergencia en Chile y en el mundo. Nunca en la historia se ha ofrecido tal variedad de plataformas para una misma enfermedad infecciosa, demostrando que el desastre pandémico pudo empujar el avance de desarrollos científico-tecnológicos que se encontraban ad-portas de alcanzar el mercado farmacéutico. Es así como a la fecha, en Chile, han recibido aprobación para uso de emergencia, vacunas basadas en la estrategia convencional constituida por virus completo inactivado (Coronavac de Sinovac), en vectores adenovirales que codifican para la proteína S (ChAdOx1 nCOVID-1 de Astrazeneca, Convidencia de Cansino y Jansen de Johnson y Johnson) y nanopartículas lipídicas que transportan el mRNA que codifica para la misma proteína $S$ (BNT162b mRNA de Pfizer/Biontech)(Chung et al., 2021).

Las primeras vacunas que obtuvieron autorización para uso de emergencia en nuestro país requerían esquemas de inmunización en dos dosis, espaciadas por 21 ó 28 días. Sin embargo, desde abril a la fecha se han incorporado dos vacunas que se administran en una sola dosis (Convidencia de Cansino y Jansen de Johnson y Johnson) que vienen a disminuir la carga logística de sus predecesoras ante el desafío tácito que significa vacunar al mayor porcentaje posible de la población en todos los rincones del territorio y en el menor tiempo posible.

Una gran preocupación surgió debido a una asociación, de bajísima frecuencia, con eventos trombóticos ocurridos principalmente en personas que recibieron las vacunas ChAdOx1 nCOVID-1 de Astrazeneca y Jansen de Johnson y Johnson. Algunos países suspendieron temporalmente la vacunación y en Chile se optó por restringir la población receptora a hombres de todas las edades y mujeres sobre 55 años (MINSAL a, 2021). Este efecto se denominó como vaccine induced immune thrombotic thrombocytopenia (VITT, por sus siglas en inglés), o thrombosis with thrombocytopenia syndrome (TTS, por sus siglas en inglés). Se trata de personas saludables que experimentan trombosis en localizaciones atípicas como venas cerebrales o del lecho esplénico a las semanas siguientes de recibir la primera dosis de la vacunación. El síndrome tiene 3 características principales: a) propensión a trombosis cerebral o esplénica, b) datos de laboratorio consistentes con coagulopatía extensiva y anticuerpos contra el factor plaquetario 4 (PF4, por sus siglas en inglés) y c) resultados inciertos. Si se detecta oportunamente es posible su tratamiento con anticoagulantes distintos a la heparina (Arepally \& Ortel 2021). En Chile, luego de reportarse un caso de trombosis en un hombre de 31 años, el MINSAL, ha decidido restringir la inmunización con esta vacuna a personas de más de 45 años (MINSAL a, 2021). Además, aquellos hombres menores a 45 años que recibieron la primera dosis de ChAdOx1 nCOVID-1, recibirán una segunda dosis de la vacuna BNT162b mRNA de Pfizer/ Biontech (MINSALb, 2021). Sobre esto último, podría levantarse una cierta preocupación, sin embargo, el estudio CombiVacS enroló 663 voluntarios que habían recibido la vacuna de Astrazeneca. En este, dos tercios, seleccionados aleatoriamente, reciben su segunda dosis de la vacuna Pfizer al menos ocho semanas después de la primera dosis. Se ha observado en estos pacientes un incremento en la producción de anticuerpos específicos inactivantes para el virus en pruebas de laboratorio. Los voluntarios que sirvieron como controles de este estudio y no recibieron el refuerzo, no experimentaron alza en sus títulos de anticuerpos específicos para SARS-CoV-2 (Callaway, 2021). Por otro lado, un ensayo similar llevado a cabo en Reino Unido llamado Com-COV informó que las tasas de efectos adversos-no graves-conocidos para las vacunas fueron más altas en los pacientes que recibían esquemas heterólogos de vacunación (Callaway, 2021).

(1) Escuela de Química y Farmacia y Centro de investigación Farmacopea Chilena, Facultad de Farmacia, Universidad de Valparaíso. Valparaíso, Chile.

(2) Facultad de Medicina Pontificia Universidad Católica de Chile. Santiago, Chile.

(3) Clínica Alemana de Santiago y Facultad de Medicina, Clínica Alemana-Universidad del Desarrollo. Santiago, Chile.

(4) Oncología Médica, Clínica Indisa. Oncología Médica, Universidad de Chile. Santiago, Chile.

(5) Oficina de Apoyo a la Investigación Clínica. Hospital Clínico Universidad de Chile.

(6) Instituto de Farmacia, Facultad de Ciencias, Universidad Austral de Chile. Valdivia, Chile.

(7) Facultad de Medicina. Clínica Alemana, Universidad del Desarrollo. Santiago, Chile.

Autora de correspondencia: caroline.weinstein@uv.cl 
El impacto que tendrá la vacunación en la población infantil sobre el curso de la pandemia tendrá que ser determinado a posteriori, sin embargo, no se puede negar que, aunque con menores consecuencias, la enfermedad puede afectar a esta población y que en casos de muy baja frecuencia puede tener consecuencias graves y hasta fatales por el desarrollo del Pediatric Inflammatory Multisystemic Syndrome (PIMS, por sus siglas en inglés) (Whittaker et al., 2020). Según una revisión sistemática y meta análisis aceptado para ser publicado, los pacientes pediátricos son; asintomáticos en un $20 \%$, y presentan sintomatología leve en un 33\%, moderada en un $51 \%$, severa en un $7 \%$, crítica en un $5 \%$; y muerte en un $0 \%$ (Cui et al., 2021). Por otra parte, una revisión narrativa sistemática que se enfocó en la población de menos de 20 años, informó que el rango de asintomáticos varió entre un 14,6 y 42\%, la presencia de fiebre se encontró entre un 46 a 64,2\%, la presencia de tos entre un 32 a 55,9\% y otros síntomas estuvieron presentes en menos de un 10 a 20\% de la población estudiada (Viner et al., 2020). Además, los expertos internacionales han afirmado que abrir los establecimientos educacionales, sin las medidas de mitigación de riesgo de infección, puede traer graves consecuencias en el avance de la pandemia (Gurdasani et al., 2021). En consecuencia, la extensión de la indicación de la vacuna BNT162b mRNA de Pfizer/Biontech al grupo etario de 12 a 16 años es una noticia positiva. También es alentador saber que la compañía biotecnológica Sinovac, también se encuentra desarrollando estudios clínicos en población pediátrica (ClinicalTrials.gov,a, 2021).

Con respecto a la emergencia de variantes del SARS-CoV-2 (actualmente denominadas por letras griegas, en vez de asociadas a la localización geográfica en que se identificaron por primera vez), que aparecen debido a mutaciones durante su proceso de replicación, y se denominan de preocupación porque podrían escapar de la capacidad neutralizante inducida por las vacunas, acrecienta el temor general a una pandemia sin fin. Una reciente publicación informa sobre la disminución de la capacidad neutralizante in vitro de anticuerpos generados por diversos tipos de vacunas ante las variantes alfa, beta y gama (Abdool Karim \& de Oliveira, 2021). Sin embargo, no se analiza si la inmunidad celular mediada por linfocitos auxiliares y citotóxicos, que son estimulados por las distintas vacunas, está reducida ante las variantes. También hay que considerar que las vacunas basadas en mRNA son bastante flexibles y pueden prontamente ser ajustadas para las nuevas variantes, tal como las vacunas contra la influenza se van ajustando a la variación del virus debido a sus mutaciones. También, es importante considerar que aún se están desarrollando y están en diversas fases de estudio vacunas contra SARS-CoV-2 y algunas aparentemente son más eficaces contra las variantes de preocupación. Sin embargo, es crucial comprender que el riesgo de aparición estas variantes que escapan a la acción de las vacunas en un período de tiempo determinado es función de la incidencia de la infección. En consecuencia, la reducción de casos no solo es primordial para disminuir la presión asistencial, sino que también lo es para bajar el riesgo de aparición de variantes resistentes a las vacunas (Thompson et al., 2021).

Finalmente, existen otras plataformas en evaluación para el desarrollo de vacunas, que resultan desafiantes y promisorias, tales como por ejemplo el uso de células presentadoras de antígeno pulsadas con péptidos del virus (Chung et al., 2021). También nuevas vías de administración que podrán alivianar la carga de los sistemas de salud y resultar más amables para los pacientes, tal como la vía intranasal. Esto último, además, podría mejorar el control pandémico si se considera que la vía intramuscular, aplicada en todas las vacunas en uso a la fecha, generan inmunidad sistémica y a nivel del tracto respiratorio inferior, a diferencia de las vacunas administradas por la vía intranasal que generan también protección inmunológica en el tracto respiratorio superior, además de la inmunidad del tracto respiratorio inferior y sistémica. De esta manera, muy importantemente, este tipo de vacunas prevendría la eliminación viral a través de la mucosa nasal, evitando la transmisión del virus entre las personas (Hassan et al., 2020). Lamentablemente, el estado de desarrollo de vacunas que utilizan plataformas basadas en vector adenoviral, virus respiratorio sincisial, Newcastle disease virus o el propio SARS-CoV-2 atenuado, administradas por la vía intranasal, recién se encuentran a nivel de ensayos clínicos fase I (Clinicaltrials.gov, b, 2021).

En conclusión, podemos decir que al enfrentar esta pandemia hemos aprendido una lección trascendental y es que las estrategias que hoy pueden parecer ciencia ficción mañana pueden estar a nuestra disposición para el tratamiento y prevención de enfermedades. Además, también hemos adquirido mayor conciencia de que todo producto terapéutico posee potenciales eventos adversos que no han sido pesquisados en los ensayos clínicos y que por ende la farmacovigilancia cobra una vital importancia en la validación de cualquier herramienta utilizada para mantener y recuperar la salud.

\section{Conflictos declarados por los autores}

La Dra Viviana Noriega participó en los ensayos clínicos para la vacuna ChAdOx1 nCOVID-1 de Astrazeneca

\section{Referencias}

Abdool Karim SS \& de Oliveira T.(2021) New SARS-CoV-2 Variants - Clinical , Public Health , and Vaccine Implications. New England Journal of Medicine, 384,1866-1868. 
Arepally GM \& Ortel TL. (2021). Vaccine-Induced Immune Thrombotic Thrombocytopenia (VITT): What We Know and Don't Know" Blood. Accedido en https://reader.elsevier.com/reader/sd/pii/ S0006497121011472?token=75CAAA5D1C05184F7FE1C9B0716D76387BB0540D62A291AF4012D412D2D245967564F00A689FD3CEE5651B9B1CD46F45\&originRegion=us-east-1\&originCreation=20210623154745 el 19 de junio de 2021.

Callaway E. (2021). Mixing COVID Vaccines. Nature 593, 491.

Chung JY, Thone MN \& Kwon YJ.(2021) COVID-19 vaccines: The status and perspectives in delivery points of view. Advanced Drug Delivery Reviews 170, 1-25.

ClinicalTrials.gov, a. Safety of an Inactivated SARS-CoV-2 Vaccine (CoronaVac) Children and Adolescents. Accessed June 20, 2021. Accedido en https://www.clinicaltrials.gov/ct2/show/NCT04884685 el 20 de junio de 2021.

Clinicaltrials.gov, b. Clinical Trials Intranasal COVID-19 Vaccines. Accedido en https://www.clinicaltrials.gov/ct2/results?cond=Covid19+vaccine\&term=intranasal\&cntry $=\&$ state $=\&$ city $=\&$ dist el 20 de junio de 2021.

Cui X, Zhao Z, Zhang T, Guo W, Guo W, Zheng J, Zhang J, Dong C, Na R, Zheng L, Li W, Liu Z, Ma J, Wang J, He S, Xu Y, Si P, Shen Y \& Cai C. (2021). A Systematic Review and Meta-Analysis of Children with Coronavirus Disease 2019 (COVID-19). Journal of Medical Virology 93, 1057-1069.

Gurdasani D, Alwan NA, Greenhalgh T, Hyde Z, Johnson L, McKee M, Michie S, Prather KA, Rassmusen SD, Reicher S, Roderick P \& Ziauddeen H. (2021). School Reopening without Robust COVID-19 Mitigation Risks Accelerating the Pandemic. The Lancet $397,1177-78$.
Hassan AO, Kafai NM, Dmitriev IP, Fox MJ, Smith BK, Harvey IB, Chen RE, Winkler ES, Wessel AW, Case JB, Kashentseva E, McCune BT, Bailey AL, Zhao H, VanBlargan LA, Dai Y-N, Ma M, Adams LJ, Shrihari S, Danis JE, Gralinski LE, Hou YJ, Schäfer A, Kim AS, Keeler SP, Weiskopf D, Baric RS, Holtzman MJ, Fremont DH, Curiel DT \& Diamond MS. (2020). A Single-Dose Intranasal ChAd Vaccine Protects Upper and Lower Respiratory Tracts against SARS-CoV-2. Cell 183,169-184.

MINSAL, a. División Prevención y Control de Enfermedades Departamento de Inmunizaciones Subsecretaría de Salud Pública (2021). Actualización de uso vacuna de Laboratorio Astrazeneca (CHADOX1-s). Accedido en https://www.minsal.cl/wp-content/ uploads/2021/06/Actualizaci\%C3\%B3n-uso-de-vacuna-laboratorio-AstraZeneca-\%E2\%80\%93-ChAdOx1-S.pdf el 18 de junio de 2021.

MINSAL, b. División Prevención y Control de Enfermedades Departamento de Inmunizaciones Subsecretaría de Salud Pública (2021). Esquema heterólogo Astrazeneca/Pfizer para completar vacunación de menores de 45 años que recibieron $1^{\circ}$ dosis de astrazeneca. Accedido en https://www.minsal.cl/wp-content/uploads/2021/06/ ESQUEMA-ASTRAZENECA-2.pdf el 18 de junio de 2021.

Thompson RN, Hill EM. \& Gog JR. (2021). SARS-CoV-2 Incidence and Vaccine Escape. Lancet Infectious Diseases 13, 913-914.

Viner RM, Ward JL, Hudson LD, Ashe M, Patel SV, Hargreaves D. \& Whittaker E. (2020). Systematic Review of Reviews of Symptoms and Signs of COVID-19 in Children and Adolescents. Archives of Disease in Childhood 0, 1-6.

Whittaker E, Bamford A, Kenny J, Kaforou M, Jones CE, Shah P, Ramnarayan P, Fraisse A, Miller O, Davies P, Kucera F, Brierley J, McDougall M, Carter M, Tremoulet A, Shimizu C, Herberg J, Burns JC, Lyall H, Levin M. \& PIMS-TS Study Group and EUCLIDS and PERFORM Consortia.(2020). Clinical Characteristics of 58 Children With a Pediatric Inflammatory Multisystem Syndrome Temporally Associated With SARS-CoV-2. JAMA 324, 259-269. 